\title{
Time-Varying Harmonic Analyzer Prototype
}

\author{
Diego F. Fabri, Carlos H. N. Martins, Leandro R. M. Silva, Carlos A. Duque, Member, IEEE, Paulo F. \\ Ribeiro, Fellow, IEEE, Augusto S. Cerqueira.
}

\begin{abstract}
This paper presents a time-varying harmonic analyzer based on the Sliding Window DFT implemented on a DSP platform. The harmonic decomposition is carried out using the TMS320F28027 digital signal processor, and the samples of the decomposed signal are sent to a PC based computer using serial interface. Several real cases are analyzed, revealing the time-varying behavior of the harmonic component. Different from commercial harmonic analyzer, the prototype presented in this paper uses a different way to visualize the harmonic components, based on time decomposition approach. This new visualization of time-varying harmonics might help engineers and researchers to develop new insights in the transient mechanisms of waveform distortions and possible new applications for power quality monitoring, control and protection.
\end{abstract}

Index Terms-harmonic decomposition, time-varying harmonics, Recursive DFT, DSP.

\section{INTRODUCTION}

$\mathbf{H}$ ARMONICS, by the classical definition, are a steady state components where the waveform to be analyzed is assumed to be periodic. The most common techniques used in harmonic analysis are based on the Fast Fourier Transform - a computationally efficient implementation of the Discrete Fourier Transform (DFT). Usually a bar graph or a magnitude response of the windowing signal is generated in order to evaluate its spectral content. The information in this kind of plot is useful when the signal under analysis is stationary, and when the leakage effect can be minimized, otherwise the information extracted from this graph can lead to misinterpretation.

The development of electronic devices and the spread of non-linear loads have increased the harmonic distortion in power systems. Besides this, the new non-linear loads, due to their dynamic nature, inject into the system time-varying components [1].

Several approaches have been proposed in recent years to improve the accuracy of spectral estimation in time-varying conditions. These include the Kalman filter based analyzer [1], adaptive notch filter [2], the Phase-Locked Loop (PLL) [3] [4] and others. Each one of these methods have advantages and disadvantages, and the search for better techniques continue in

This work was supported in part by CNPq, CAPES and FAPEMIG (Brazilian research agencies). Diego F. Fabri is with Federal University of Juiz de Fora, Juiz de Fora, MG, Brazil, and PSCOPE/UFJF (diego.fabri@engenharia.ufjf.br), Carlos $\mathrm{H}$ N. Martins (chnmartins@yahoo.com.br) and Leandro R.M. Silva (leandro.manso@engenharia.ufff.br) are with Federal University of Juiz de Fora, Juiz de Fora-MG, Brazil, and PSCOPE/UFJF. Carlos A. Duque (carlos.duque@ufjf.edu.br), is with Electrical Engineering Dept., UFJF, Juiz de Fora MG, Brazil and Paulo F. Ribeiro (pfribeiro@ieee.org) is with Calvin College, Grand Rapids, Michigan, USA, Electrical Engineering Dept., UFJF, Juiz de Fora MG, Brazil, and Technical University of Eindhoven, Eindhoven, Netherlands. the signal processing area. However, all of these methods have in common the way that the information is presented to the user: they are concerned to show the time-varying behavior of the magnitude and phase estimation of each pre-defined component in the estimator model. They are in fact amplitude and phase estimators.

Another way to analyze time varying signals is using the concept of decomposition. Decomposition techniques are concerned with the way that the original signal can be split in individual components, including harmonics, interharmonics, sub-harmonics, etc. Usually, signals decomposition is carried out in the time domain, in a way that the time-varying behavior of each harmonic component can be observable. This subject is important in power quality analysis for different reasons, including analysis of loads behavior, failure detection, and pattern recognition of events, among others. The main techniques used in signal decomposition are wavelet transforms [5] [6], filter banks decomposition approach [7]-[9] and sliding window recursive DFT (SWRDFT) [10].

The Wavelet Transform approach has the disadvantage to decompose the signal in components that may not have physical meaning in power system terms.

Filter banks approach is able to decompose the signal in its time varying harmonic components; however in the current stage of research they have complex structure to be implemented in real time and their transient response are too long.

SWRDFT approach presented in [10] is able to decompose the signal in its time-varying harmonic component and has the following advantages: low computational complexity and a single cycle of transient response.

This paper presents a prototype of Time Varying Harmonic Analyzer. The core of the analyzer is the SWRDFT approach presented in [10] and implemented in low cost Digital Signal Processor (TMS320F28027). The paper presents an overview of the hardware and software implementation, a theoretical discussion and some waveforms decomposition. The main contribution of this paper is the waveform decomposition plot, since this method of visualization is rarely used by professionals in the field of power systems. The authors believe that the Time Varying Harmonic Analyzer can become an important tool for diagnosis of power quality problems; beside this, the decomposition method introduced by the authors can boost the development of a new solution for control, protection and PQ analysis of power systems.

The paper is divided in the following sections: Section 2 presents a review of the SWRDFT approach for time varying decomposition analysis; section 3 presents the overview of the hardware and software implementation; section 4 presents several waveform decomposition acquired from real systems 
and electro-electronics equipments. Finally section 5 presents the conclusions and future works.

\section{SLIDING-Window RECURSIVE DFT}

The Recursive DFT or Sliding-Window DFT for timevarying harmonic decomposition was presented in [10]. This method is based on the rectangular form of the Fourier Series, for harmonic decomposition, and a digital sine-cosine generator for reconstruction. This section presents a brief description of this technique which constitutes the fundamental algorithm of the time-varying harmonic analyzer proposed in this paper, more information can be found in [10]-[12]. Furthermore the theoretical analysis of the behavior of the SWRDFT and a simple way to identify which harmonic decomposition is or not a time-varying component is introduced.

Given a signal $x(n)$, this can be reconstructed through the quadrature terms, $Y_{C_{h}}$ and $Y_{S_{h}}$ of the Fourier Series (1), where $h$ is the harmonic. These quadrature components can be easily obtained by recursive equations (2),

$$
\begin{array}{r}
x(n)=a_{0}+2 \sum_{h=1}^{H} Y_{C_{h}}(n) \cdot \cos \left(w_{h} n\right) \\
-Y_{S_{h}}(n) \cdot \sin \left(w_{h} n\right)
\end{array}
$$

where,

$$
\begin{aligned}
Y_{C_{h}}(n)= & Y_{C_{h}}(n-1) \\
& +(x(n)-x(n-N)) \cdot \cos \left(w_{h} n\right) \\
Y_{S_{h}}(n)= & Y_{S_{h}}(n-1) \\
& -(x(n)-x(n-N)) \cdot \sin \left(w_{h} n\right)
\end{aligned}
$$

and $w_{h}$ is the $h$-th harmonic frequency.

The sine and cosine terms present in (1) can be obtained by a digital sine-cosine generator, it can be implemented through state equations (3), where $s_{1}(n)$ is a sine function and $s_{2}(n)$ a cosine function.

$$
\begin{aligned}
{\left[\begin{array}{l}
s_{1}(n) \\
s_{2}(n)
\end{array}\right]=} & {\left[\begin{array}{cc}
\cos \left(w_{h} n\right) & \cos \left(w_{h} n\right)+1 \\
\cos \left(w_{h} n\right)-1 & \cos \left(w_{h} n\right)
\end{array}\right] } \\
& \cdot\left[\begin{array}{c}
s_{1}(n-1) \\
s_{2}(n-1)
\end{array}\right]
\end{aligned}
$$

The structure for extracting the $h$-th harmonic can be seen in Fig. 1. As can be seen, the sine and cosine generated by digital generator are both used in the decomposition and harmonic reconstruction.

In adopting this sine-cosine generator, both, the decomposition and the reconstruction tasks, can run parallel to each other, according to Fig. 1.

Of course, to extracting $N$ harmonics it is necessary to employ $N$ structures as shown, but there are some advantages when using it, such as:

i low computational effort, suitable for real time decomposition implementation;

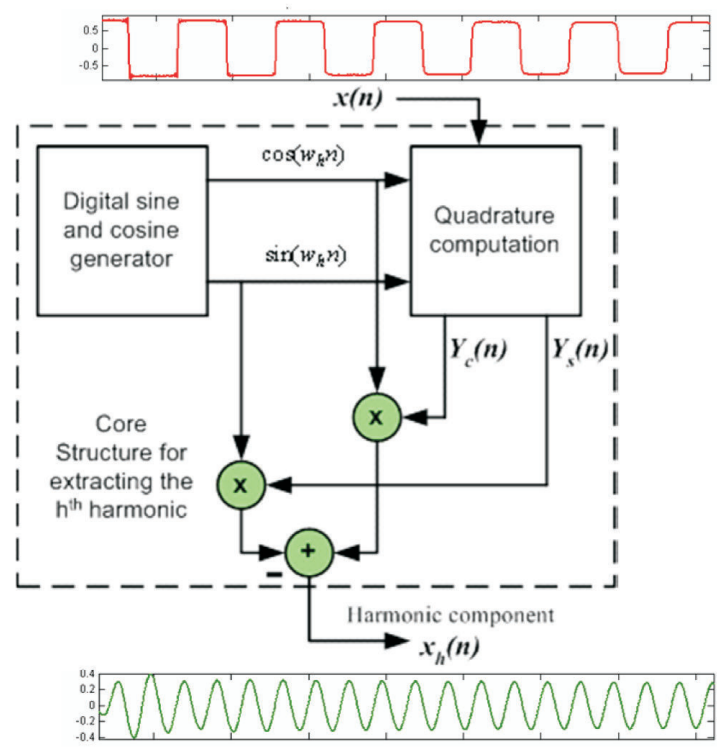

Fig. 1. Core Structure For Extracting the $h^{\text {th }}$ Harmonic

ii no phase delay;

iii transient time equal to the sliding window width. For window of one cycle, the convergence is reach after one cycle.

\section{A. Theoretical Analysis}

To explore some advantages of time varying harmonic decomposition and go toward to the possibility of new interpretation brought by the analyzer, two theoretical cases are considered. They are represented by equations (4a) and (4b), where (4a) represents an interharmonic added to the fundamental component and (4b) represents a fundamental component being modulated by a sinusoid function. In these equations $f_{0}$ represents the fundamental frequency $(60 \mathrm{~Hz}), f_{i}$ and $f_{m}$, respectively the interharmonic and the modulating frequency, $A_{i}$ and $A_{m}$ the interharmonic and modulating amplitude and $T_{s}$ the sampling time.

$$
\begin{gathered}
x_{1}(n)=\cos \left(2 \pi \cdot f_{0} \cdot T_{s} \cdot n\right)+A_{i} \cdot \cos \left(2 \pi \cdot f_{i} \cdot T_{s} \cdot n\right) \\
x_{2}(n)=\left(1+A_{m} \cdot \cos \left(2 \pi \cdot f_{m} \cdot T_{s} \cdot n\right)\right) \cdot \cos \left(2 \pi \cdot f_{0} \cdot T_{s} \cdot n\right)
\end{gathered}
$$

Figure 2 portrays the waveform of $x_{1}(n)$ and $x_{2}(n)$, respectively.

Figure 3 shows the harmonic decomposition of the signal $x_{1}(n)$. Note that the interharmonic of $174 \mathrm{~Hz}$ spillover to the adjacent harmonics, second and four. The spillover is due the same effect well known as leakage in spectral analysis. This is because the present method uses as decomposition filter the same as used in DFT analysis. However the decomposition approach reveals information not clear in the FFT approach. For example the FFT of the signal $x_{1}(n)$, using 12 cycles, as recommended by IEC61000-4-30, is presented in Fig. 4. Note that from the spectral analysis we cannot affirm what frequency are presents near the third harmonic. On the other 

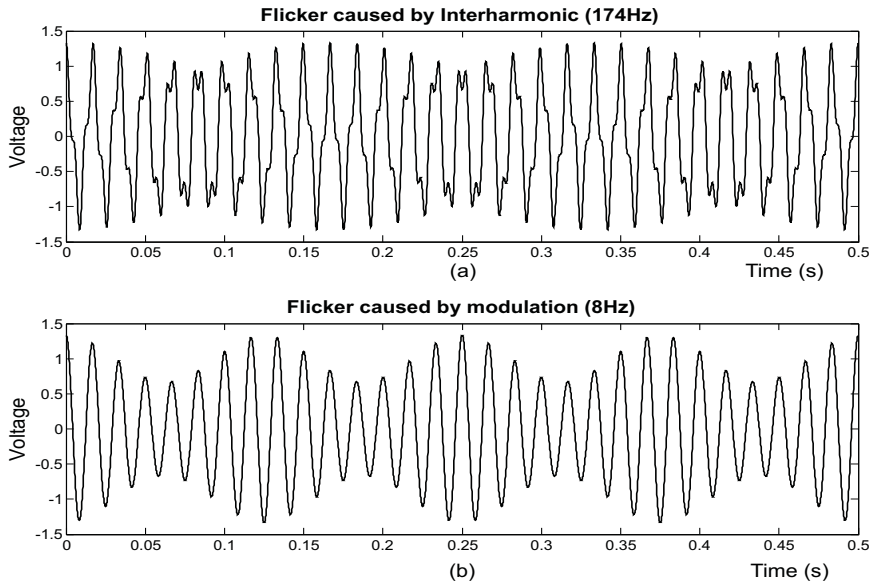

Fig. 2. (a) $x_{1}(n)$ waveform (b) $x_{2}(n)$ waveform
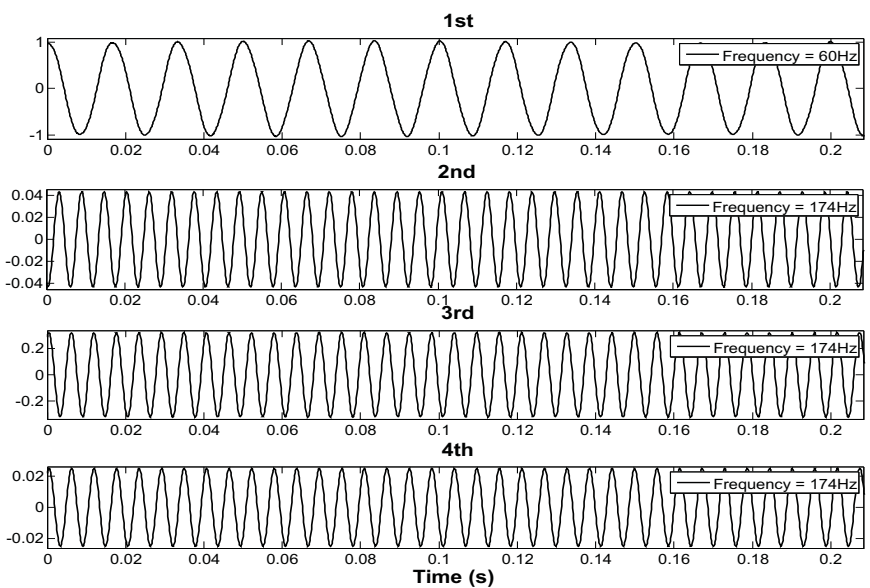

Fig. 3. Interhamonic decomposition

hand the signals observed at the second and fourth harmonic decomposition plot (Fig. 3) have the frequency of $174 \mathrm{~Hz}$, the same that appears on the third harmonic plot. It is easy to conclude using the decomposition approach that the signal contains an interharmonic and interharmonic spills over to adjacent frequencies. The frequency estimation is performed using the algorithm on [13].

Figure 5 shows the harmonic decomposition for signal $x_{2}(n)$. As we can see the signal that appears at first harmonic can be considered as a time-varying harmonic. In fact $x_{2}(n)$ can be written in a compact form $x_{2}(n)=A(n) \cdot \cos \left(w_{0} n\right)$. Of course that the signal is composed by the sum of the fundamental signal plus two interharmonic close to it, inside the same band. In this work we are using the following definition for time-varying harmonic: a time varying harmonic is that one that can be written in the general form

$$
x_{h}(n)=A_{h}(n) \cdot \cos \left(h \cdot w_{0}+\theta(n)\right)
$$

Of course it is not easy from the plot decomposition verify if the above expression is true or not, however the plot shows that the harmonic under analysis has similar behavior as time varying.

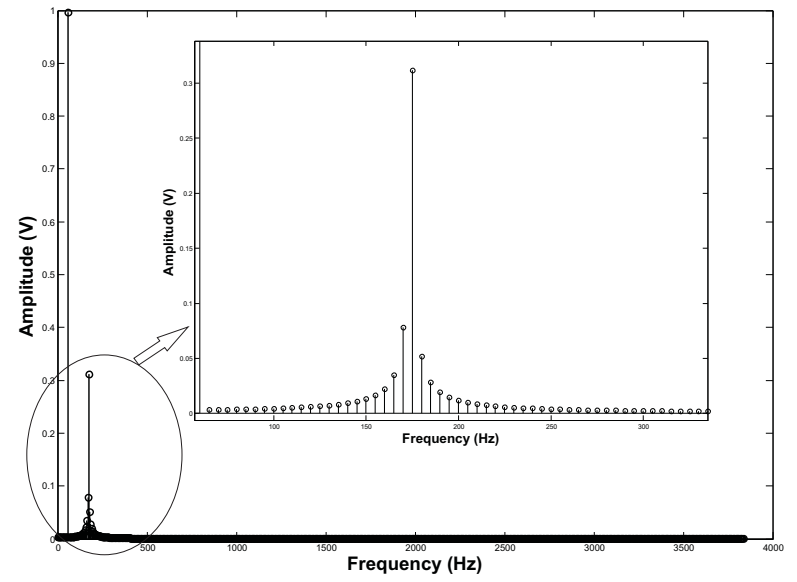

Fig. 4. Interharmonic FFT

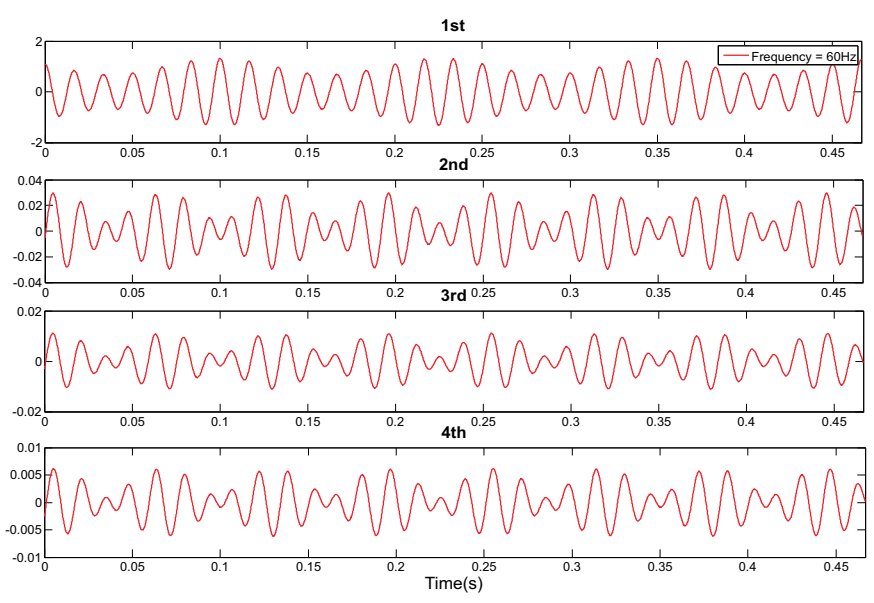

Fig. 5. Modulation decomposition

Due the filter nature choosing for the prototype analyzer the interharmonics close the fundamental component spill over to adjacent components. The user can easily indentify that the other components are results from spillover, first because the decreasing energy on subsequent harmonics and second because the same waveform. At the moment the authors are investigating some parameters to be used together the plot to introduces extra information. For example, the authors have been using the Crest Factor as a indicative if the waveform is a pure sinusoid or not. When the decomposition is not a pure sinusoid the correspondent plot is highlight in a different color (red in this paper). This allows easy visual identification.

\section{Time-VARying AnAlyzer Structure}

The system is composed by a dedicated hardware and firmware. Hardware consists in conditioning, acquisition and signal processing systems. Firmware is the DFT SlidingWindow algorithm embedded on DSP platform.

\section{A. Hardware}

Hardware can be divided into three parts:

- Signal conditioning system and Anti-Aliasing Filter

- Digital Signal Processor (DSP) 


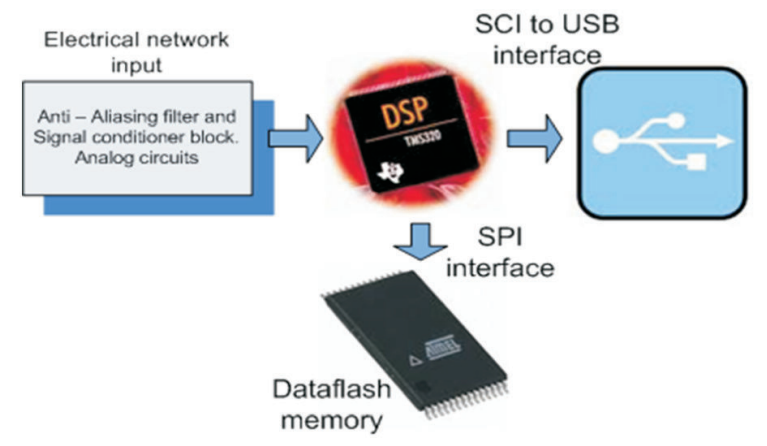

Fig. 6. Overview of time-varying analyzer hardware

- External Memory Device (dataflash).

The signal conditioning system consists of step-down voltage transformer, current probe and analog circuit to signal excursion control. The anti-aliasing filter is sixth-order Butterworth with cutoff frequency of $1600 \mathrm{~Hz}$. This cutoff frequency is chosen because the present implementation of the analyzer was designed to analyze up to the 25th harmonic $(1500 \mathrm{~Hz})$.

All the signal processing and the harmonic decomposition are done by DSP TMS320F28027 [14] from Texas Instruments. This processor has a 12 bits Analog-Digital Converter with $0.8 \%$ of error, 32-bit Timers, a Serial Communication Interface (SCI) and a Serial Peripheral Interface (SPI).

The analog-digital converter operates in continuous acquisition mode (free-running mode), but a new sample is read only every timer interrupt, which occurs every 130us. That is, the system works with a sampling frequency of $7680 \mathrm{~Hz}$. The serial communication interface is used to perform the communication between the DSP and the PC, via RS-232 interface, and the serial peripheral interface is used to communicate DSP with an external memory device.

A $64 \mathrm{Mb}$ Data-Flash memory is used as an external memory device. This device allows the storage the waveforms of harmonic decomposition, allowing for later visualization of these components.

The overview of system hardware can be seen in Figure 6 .

\section{B. Firmware}

The firmware is based on sliding-window DFT algorithm. That algorithm is recursive, i.e., operates sample-by-sample, at each timer interrupt. The signal decomposition is computed at each new sample, through the quadrature components and the digital sine and cosine.

The reconstruction $x_{h}(n)$ at instant $n$ is stored in an output buffer. This buffer of length 128 , when is full, is transferred to the external memory buffer. The control of this process is accomplished through control flags. After the memory is full, data is transferred through the SCI to PC, where the data are displayed.

The program was written in $\mathrm{C}$ language and using a fixed point arithmetic using the IQmath library [15] available from Texas Instruments.

The overview of firmware can be seen in Figure 7 .

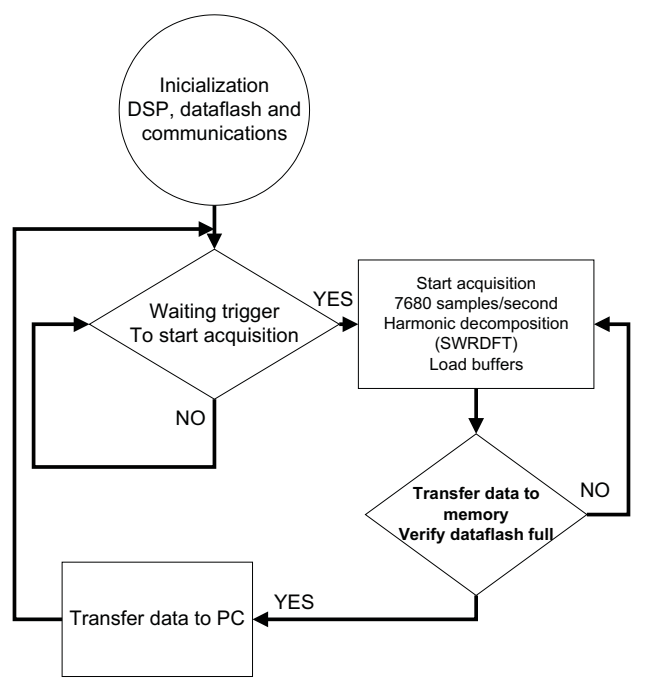

Fig. 7. Overview of time-varying analyzer firmware

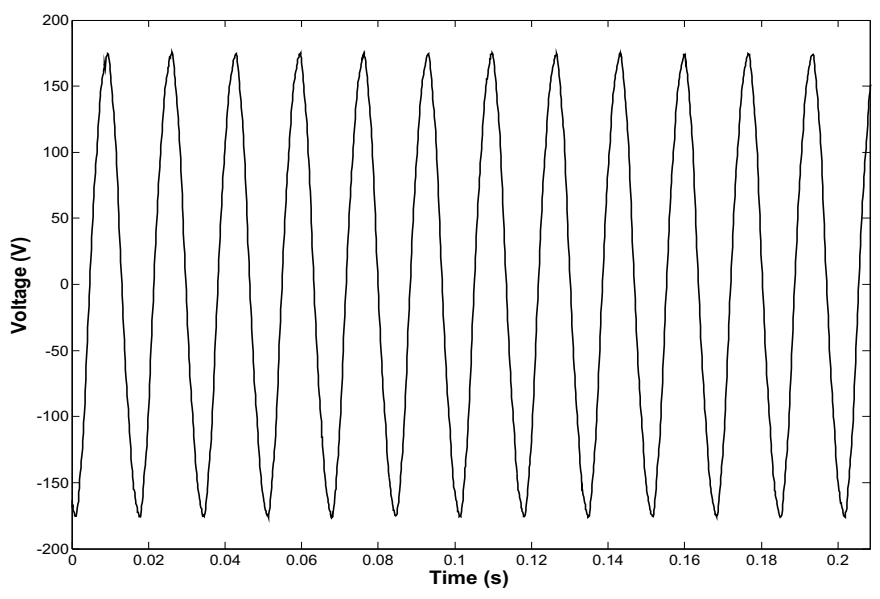

Fig. 8. LAPTEL voltage

\section{Practical Results}

In order to analyze the performance of the prototype analyzer, several distorted signals were acquired and processed by the system. This section presents the time varying harmonic of the following cases:

a Voltage waveform acquired at LAPTEL laboratory;

b Microwave oven (Eletronic Device/Appliance);

c Induction Motor with frequency inverter.

\section{A. Voltage waveform acquired at LAPTEL/UFJF}

The LAPTEL (Telecommunication and Signal Processing Laboratory) is composed basically by several computers, electronic equipments and fluorescent lamps. Figure 8 shows the voltage waveform obtained from phase A $(127 \mathrm{~V})$.

Figure 9 shows the harmonic decomposition of LAPTEL/UFJF voltage. Note that the time-varying behavior of harmonic components. The amplitude of these components shows variations, even small, over time. This behavior is automatically detected through the crest factor. It is also 


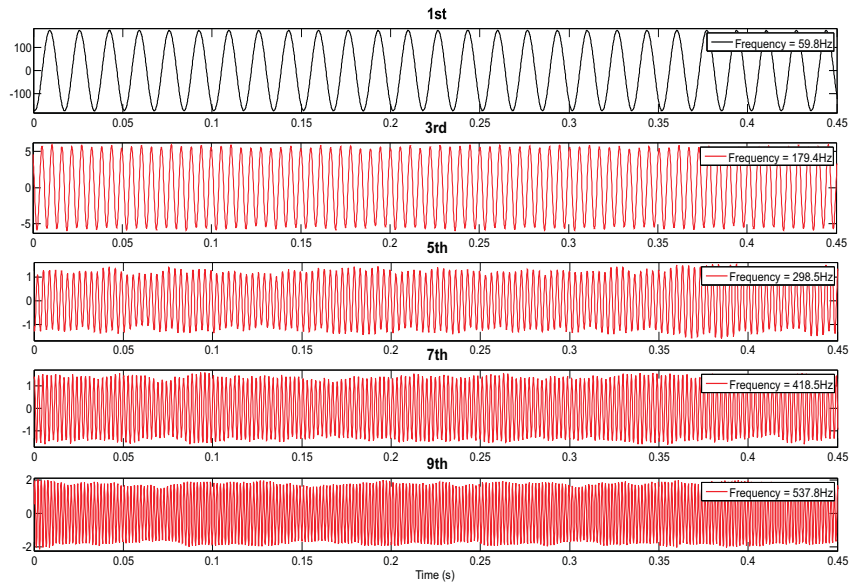

Fig. 9. LAPTEL voltage decomposition

important to note that the harmonics shown in Fig. 9 are the most representative, or those with the highest energy.

The presence of these time-varying components can be explained by the large amount of electronic equipment at the place where the measurement was performed, as computers, electronic ballasts, switching power supplies and airconditioning system.

\section{B. Electronic Device/Appliance (Microwave oven)}

The microwave oven is an electronic device where the AC supply is converted into DC through a rectifier and a largevalue capacitor for current smoothing. When a nonsmoothed power supply is used, the output voltage of the inverter may not be boosted to the operating voltage of the magnetron if the power supply voltage is low. Then, the magnetron ceases to operate and distortion is generated due to stoppage of the input current [16].

The analyzed case is from a microwave oven $1450 \mathrm{~W}, 127 \mathrm{~V}$. Figure 10 shows the microwave oven input current and Fig. 11 shows the harmonic decomposition. Note that the timevarying behavior of harmonic components, highlighted in red. When the system stabilizes, some harmonic components do not exhibit more variations (black part of the signal). It is also important to note that the harmonics shown in Fig. 11 are the most representative, or those with the highest energy

It is interesting to observe the even harmonics behavior (2nd and 4th). Initially they have low amplitude compared to the fundamental component and other harmonics. However, its amplitude will grow until the moment where there is a current transition, highlighted in Figure 10. After this, even harmonics still present a time-varying behavior, but with greater amplitude than at the start of the microwave oven.

\section{Induction Motor with Frequency Inverter}

It is well known that induction motors controlled by frequency inverters introduce harmonic distortion in the system. In situations of motor start, speed variation and load change, the harmonic components show variations in their amplitude, suggesting a time-varying behavior. Thus, it becomes interesting view of each component individually in the time domain.

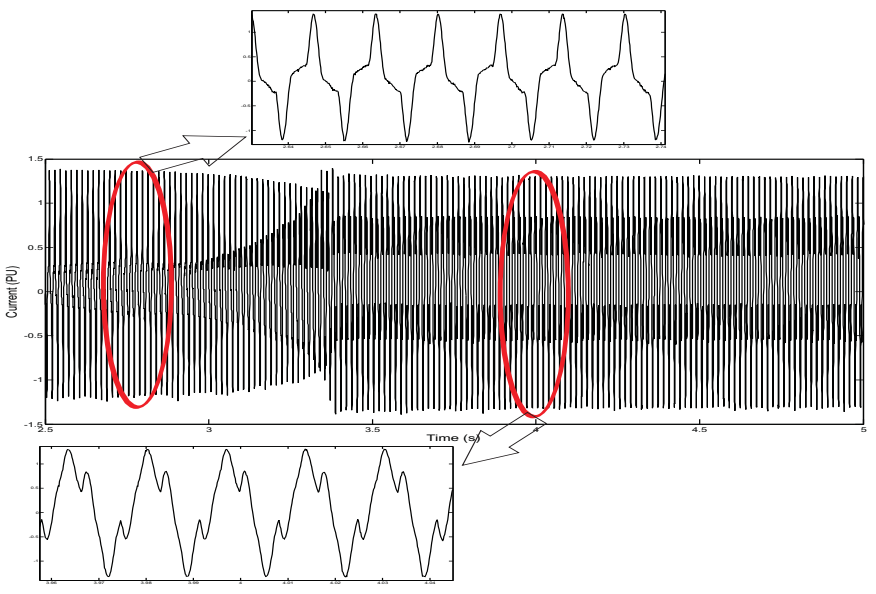

Fig. 10. Microwave oven input current

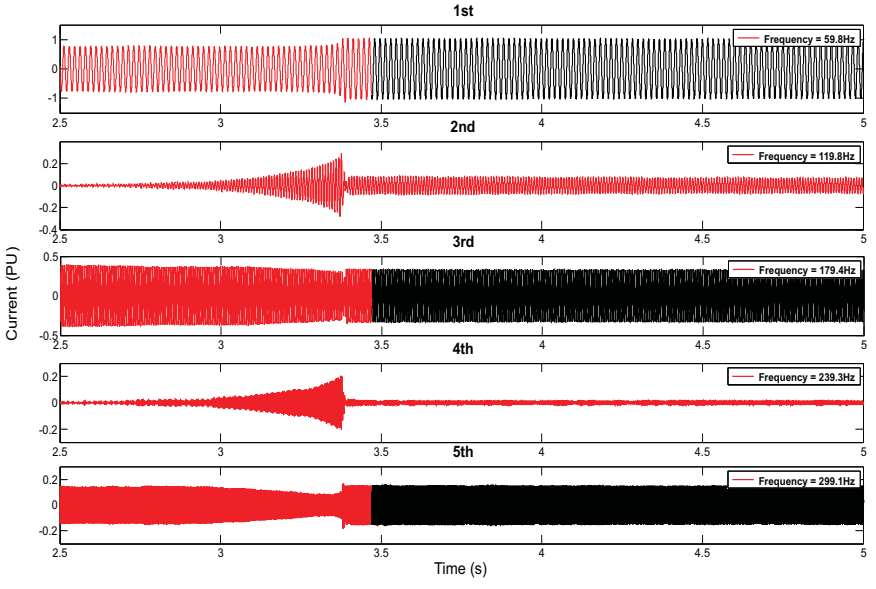

Fig. 11. Microwave oven input current decomposition

The analyzed case is a squirrel-cage induction motor (1.1HP, $220 / 380 \mathrm{~V}$ and $4.41 / 2.50 \mathrm{~A}$ ) fed by a sinusoidal PWM frequency inverter, dynamometer with torque indicator balance, bidirectional brake disc (Foucalt break) and controls for variable speed and load. A frequency inverter was monitored using the time-varying harmonic analyzer prototype. The current of the model available in the laboratory was captured and decomposed, showing to be a much distorted signal.

Figure 12 shows the harmonic decomposition for a motor starting and Fig. 13 shows the harmonic decomposition for load variation. Note that these components are much distorted as well, however due the limitation of the present version is not easy to conclude if the distortion comes from interharmonic component or spectral leakage. A new version of the time varying harmonic analyzer, that uses filter bank approach to reduce the spillover between adjacent components is being investigated by the authors. As previously mentioned the challenges are to reduce the computational complexity and the transient response of the filter banks.

\section{CONCLusions AND Future Works}

The presence of time-varying components caused by the dynamic nature of the new non-linear loads have brought the 


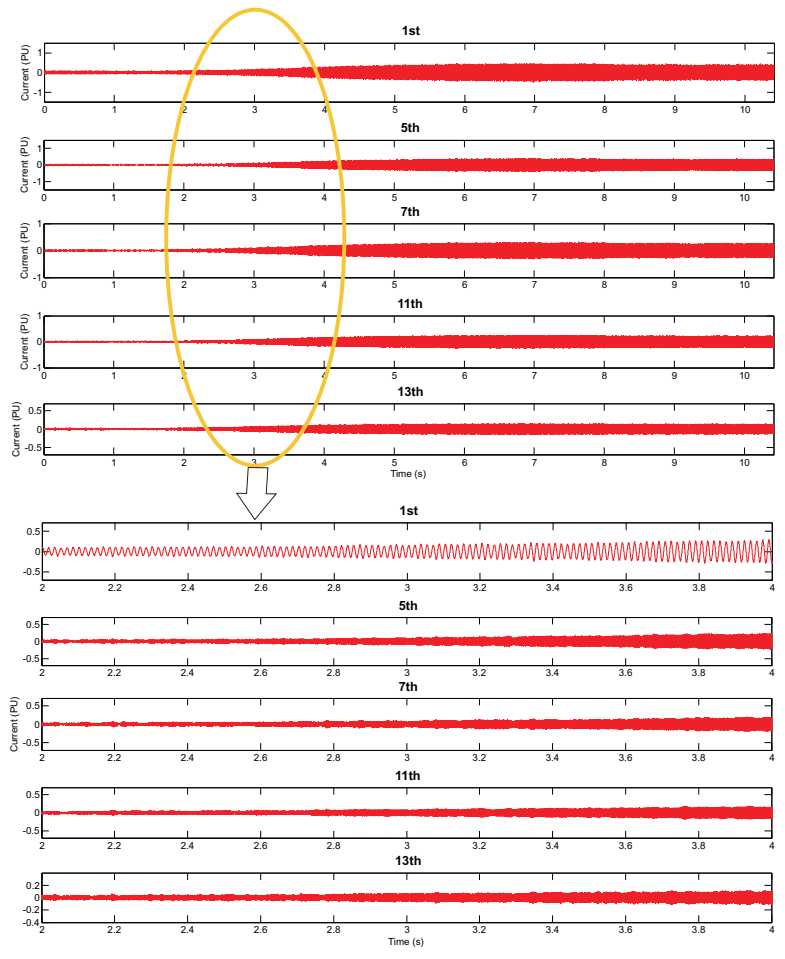

Fig. 12. Motor starting harmonic decomposition

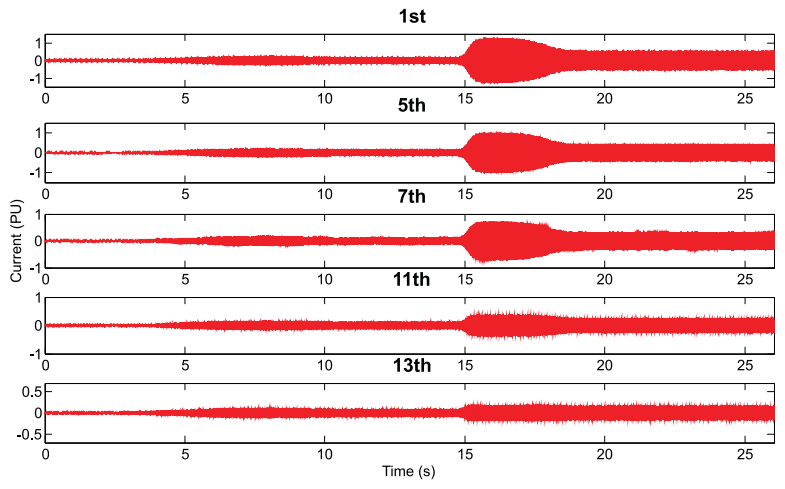

Fig. 13. Load variation harmonic decomposition

need for development of devices capable of measuring and monitoring the time-varying harmonics behavior. This new perspective led the research and development of a time-varying harmonics analyzer prototype.

This paper presents a prototype for performing time-varying harmonic analysis of single-phase systems. The prototype consists of the DSP TMS320F28027 from Texas Instruments, signal conditioning circuits and external memory devices.

The system uses the sliding-window DFT to perform the harmonic decomposition. A detailed analysis of the method is made, as well a study of harmonic decomposition in the presence of interharmonics or modulation in the fundamental component. The analysis of practical results shows the timevarying nature of harmonic components.

Some points are still being researched and developed, and eventually will be attached to the time-varying harmonics analyzer project. In the final prototype, three DSPs will be used for three-phase current and voltage analysis, and a microcontroller that will manage the entire system. The improved communication system (DSP-PC) is an important issue which also being treated, aiming to develop a system for real-time analysis of harmonic components. Next to it, we seek to improve the visualization software.

In the theoretical context, techniques for minimizing the effects of spillover have been developed (such as filter banks) and its implementation on a DSP platform is a future goal in the design of harmonic analyzer. Regarding the SWRDFT method, solutions to the errors caused by asynchronous sampling are also being investigated.

\section{ACKNOWLEDGMENTS}

The authors would like to thanks Prof. Pedro Gomes Barbosa for their support, useful and constructive suggestions.

\section{REFERENCES}

[1] S. H. Hosseini and K. Mohammadi, "Design and Implementation of a Kalman Filter-Based Time-Varying Harmonics Analyzer", Journal of Iranian Association of Electrical and Electronics Engineers, Vol.3, No.2, 2006.

[2] M. Karimi-Ghartemani, M. Mojiri and A. R. Bakhsahai, "A Technique for Extracting Time-Varying Harmonic based on an Adaptative Notch Filter", Proc. of IEEE Conference on Control Applications, Toronto, Canada, Aug. 2005

[3] J. R. Carvalho, P. H. Gomes, C. A. Duque, M. V. Ribeiro, A. S. Cerqueira and J. Szczupak, "PLL Based Harmonic Estimation", IEEE PES conference, Tampa, Florida-USA, 2007.

[4] J. R. Carvalho, C. A. Duque, M. V. Ribeiro, A. S. Cerqueira and P. F. Ribeiro, 'Time-Varying Harmonic Distotion Estimation using PLL Based Filter Bank and Multirate Processing", VII CBQEE, Aug. 2007, Brazil.

[5] P. M. Silveira, M. Steurer and P. F.Ribeiro, "Using Wavelet decomposition for Visualization and Understanding of Time-Varying Waveform Distortion in Power System", VII CBQEE, Aug. 2007, Brazil.

[6] V. L. Pham and K. P. Wong, "Antidistortion method for Wavelet Transform Filter Banks and Nonstationary Power System Waveform Harmonic Analysis", IEE Proc. Gener., Transm., Distrib., Vol 148, No.2, March 2001, pp. 117-122.

[7] H. Sun, G. H. Allen and G. D. Cain, "A new filter-bank configuration for harmonic measurement", IEEE Trans. on Instrumentation and Measurement, Vol. 45, No.3, June 1996, pp. 739-744.

[8] C.-L. Lu, "Application of DFT filter bank to power frequency harmonic measurement", IEE Proc. Gener. , Transm,. Distrib., Vol 152, No. 1, Jan. 2005, pp. 132-136.

[9] C. A. Duque, P. M. Silveira, T. Baldwin and P. F. Ribeiro, "Novel method for tracking time-varying power power harmonic distortion without frequency spillover", IEEE 2008 PES, July 2008, Pittsburgh, PA, USA.

[10] P. M. Silveira, C. Duque, T. Baldwin and P. F. Ribeiro, "Time-Varying Power Harmonic Decomposition using Sliding-Window DFT", The 13th IEEE International Conference on Harmonics and Quality of Power, Wollongong, 2008.

[11] R. Hartley and K. Welles, "Recursive Computation of The Fourier Transform”, IEEE. Int. Symposium on Circuits and Systems, Vol.3, 1990. pp. 1792-1795.

[12] Sanjit K. Mitra, Digital Signal Processing - A computer-based approach, Mc-Graw Hill 2006, 3ł Edition.

[13] Carvalho, J. R., Duque, C. A., Ribeiro, P. F. and Coury, D. V., "Algoritmo baseado na DFT para Análise Harmônica/Inter-harmônica Eficiente em Condições de Frequiência Variante". VIII Conferência Brasileira sobre Qualidade de Energia Elétrica, 2009.

[14] Texas Instrument - "Hardware Design Guidelines for TMS320F28xx and TMS320F28xxx DSCs (spraasla)" " http://focus.ti.com/lit/an/spraas1a/spraas la.pdf .

[15] Texas Instrument - "CQ28x IQmath Library - Avirtual Floating Point Engine - V.15a",

[16] Daisuke Bessyo, Kenji Yasui and Mutsuo Nakaoka, "A Method of Decreasing the Harmonic Distortion for Inverter Microwave Oven", Electronics and Communications in Japan,Part 1, Vol. 85, No. 4, 2002. 


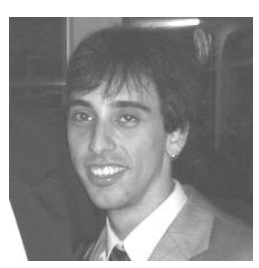

Diego F. Fabri was born in Itajubá-MG, Brazil, on July 21, 1985. He received the degree in Electrical Engineering from the Federal University of Juiz de Fora, in 2008. At this moment, he is completing his studies in the Electrical Engineering M.Sc. Program of Federal University of Juiz de Fora and act as a researcher and coordinator at Signal Processing and Computational Intelligence for Power Systems (PSCOPE) group.

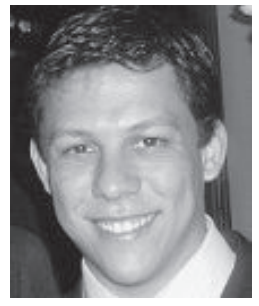

Carlos H. N. Martins was born in CongonhasMG, Brazil, on August 04, 1982. He received the degree in electronic technician from Federal technologic center of Minas Gerais (CEFET-MG), in 2001, received the degree in Electrical Engineering from Federal University of Juiz de Fora, in 2008. At this moment, he is completing his studies in the Electrical Engineering M.Sc. Program of Federal University of Juiz de Fora and act as a researcher at Signal Processing and Computational Intelligence for Power Systems (PSCOPE) group.

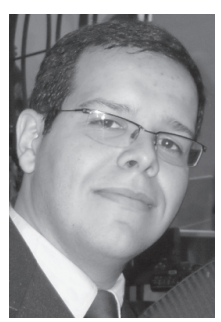

Leandro R.M. Silva was born in Valença, Brazil, on August 14, 1985. He received the degree in Electrical Engineering from Federal University of Juiz de Fora, in 2009. At this moment, he is completing his studies in the Electrical Engineering M.Sc. Program of Federal University of Juiz de Fora and act as a researcher at Signal Processing and Computational Intelligence for Power Systems (PSCOPE) group.

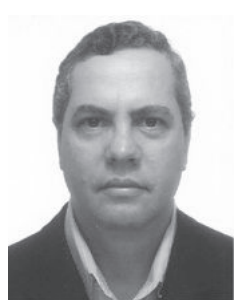

Carlos A. Duque (M'91) was born in Juiz de Fora, Brazil, in 1962. He received the B.S. degree in Electrical Engineering from the Federal University of Juiz de Fora, Brazil, in 1986, and the M.Sc. and the Ph.D.degree from the Catholic University of Rio de Janeiro, in 1990 and 1997, respectively, in Electrical Engineering. Since 1989 he is a Professor in the Engineering Faculty at Federal University of Juiz de Fora (UFJF), in Brazil. His research interests are power quality analysis, digital instrumentation and digital signal processing.

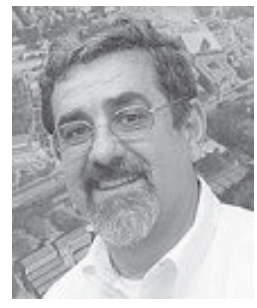

Paulo F. Ribeiro (M'78-SM'88-F'03) received a $\mathrm{BS}$ in Electrical Engineering from the Universidade Federal de Pernambuco, Recife, Brazil, completed the Electric Power Systems Engineering Course with Power Technologies, Inc. (PTI) 1, and received the Ph.D. from the University of Manchester, Manchester-UK. Presently, he is a Professor of Engineering at Calvin College, Grand Rapids, Michigan, USA. Ribeiro is active in IEEE, CIGRE and IEC working groups on power quality. He is Registered Professional Engineer in the State of Iowa
Augusto S. Cerqueira received the B.S. and D.Sc degrees in 1998 and 2002, respectively. He has been an Associate Professor at the Electrical Engineering Department of Federal University of Juiz de Fora, Brazil, since 2004 . He is been working recently with instrumentation, digital signal processing and power quality issues. 